\title{
La reglamentación europea en materia de agencias de calificación crediticia: perfiles de responsabilidad*
}

\section{Carlotta Rinaldo*}

Resumen: El presente artículo, luego de exponer brevemente el rol de la calificación crediticia en los mercados financieros, brinda una primera panorámica sobre la normativa delineada por el legislador europeo, en particular sobre aquellas normas relativas al tema de la responsabilidad civil de las agencias en caso de difusión de una calificación crediticia errónea, y pone al descubierto algunas de sus deficiencias.

Palabras clave: agencias de calificación, calificación crediticia, responsabilidad civil, mercado de capitales, Reglamento CE n. ${ }^{\circ}$ Io60/2009.

\section{European Regulation on Rating Agencies: Liability Profiles}

Авsтract: This article presents briefly the role of credit rating in the financial markets. After it provides a first overview of the legislation outlined by the European legislator, in particular on the rules on civil liability of agencies in the

Fecha de recepción: 23 de enero de 2017 . Fecha de aceptación: 22 de mayo de 2017 . Traducción al castellano de Catalina Salgado Ramírez. Título original: La regolamentazione europea in materia di agenzie di rating: profili di responsabilità. Para citar el artículo: Rinaldo, C., "La reglamentación europea en materia de agencias de calificación crediticia: perfiles de responsabilidad", Revista de Derecho Privado, Universidad Externado de Colombia, n. ${ }^{\circ}{ }^{2}$, enero-junio de 2017, 353-38г. DOI: https://doi.org/IO.I860I/OI234366.n32.I2

* Posdoctorado por la Università degli Studi di Padova. Doctorado por la Humboldt Universität zu Berlin. Investigadora del Departamento de Derecho Privado y Crítica del Derecho de la Università degli Studi di Padova, Padua, Italia. Contacto: carlotta.rinaldo@unipd.it 
event of dissemination of an erroneous credit rating. At the end, it exposes some of its shortcomings.

KEYwords: Rating agencies, credit rating, civil liability, capital markets, EC Regulation $\mathrm{r} 060 / 2009$.

Sumario. Introducción. I. La calificación crediticia (rating) en el contexto actual de los mercados financieros. II. La introducción de una reglamentación europea en materia de calificación crediticia. III. El segundo reglamento modificatorio del Reglamento CE n. ${ }^{\circ}$ Io6o/2009. Iv. Los presupuestos para una responsabilidad de las agencias de calificación frente a emisores e inversionistas. v. Algunas reflexiones al margen del remedio europeo. Bibliografía.

\section{Introducción}

Las agencias de rating o agencias de calificación, en calidad de intermediarios de información, juegan un rol de vital importancia en los mercados, esto por el hecho de representar la disponibilidad de informaciones confiables sobre los productos financieros y sus emisores uno de los pilares fundamentales para un mercado de capitales eficiente y productivo. Sin embargo, tal poder no siempre ha ido de la mano con la independencia y la profesionalidad que resultan necesarias en sujetos que gozan de tal posición. En este contexto, fue imprescindible la introducción a nivel europeo de una reglamentación específica de la actividad de las agencias, que disciplinase los aspectos que resultaron ser en la práctica los más problemáticos. En particular, en el año 2013 se introdujo un específico remedio resarcitorio, accionable por parte de los inversionistas perjudicados por una calificación (rating) incorrecta.

El presente escrito está dedicado al análisis de estas disposiciones y tiene como fin brindar una primera panorámica sobre la normativa delineada por el legislador europeo; en especial, se concentra en las normas relativas al tema de la responsabilidad civil de las agencias en caso de difusión de una calificación errónea. La calificación es un instrumento de especial importancia en la materia objeto de examen, debido al efecto disuasivo que puede tener en el comportamiento de los intermediarios financieros. Debemos acometer entonces la tarea de evaluar si esta reciente previsión de responsabilidad es capaz de cumplir un efecto general preventivo, fuera del compensatorio, al desincentivar las conductas incorrectas de las agencias, o si en cambio es dado concluir, como empiezan a subrayarlo algunos autores $^{\mathrm{I}}$, que en realidad se trata de un instrumento de mera fachada, que no modifica en modo sustancial el statu quo.

I Schroeter, Ratings - Bonitätsbeurteilungen durch Dritte im System des Finanzmarkt-, Gesellschaftsund Vertragsrechts, Tübingen, 2014, $95^{2}$ ss., esp. 954 ss. 


\section{La calificación crediticia (rating) en el contexto actual de los mercados financieros}

La crisis económica y financiera que ha marcado el último decenio ha tenido serias repercusiones en el mundo de la economía y una influencia no menos importante en el derecho de los mercados financieros. En efecto, ella ha sacado a la luz, con trágica evidencia, múltiples problemáticas que caracterizaban -y que en parte aún caracterizan- los mercados internacionales; problemáticas relativas, por una parte, a la estructura de los mercados financieros en sí mismos considerados, y por otra, a determinados mecanismos y comportamientos realizados por ciertos actores, en particular por las agencias de calificación (agencias de rating). Así las cosas, se ha generado un importante debate, también jurídico, que ha puesto en discusión la capacidad de estos actores de autorregularse de manera correcta y eficiente, y como consecuencia la convicción, bastante arraigada hasta que la crisis se presentó, según la cual no era necesaria una intervención legislativa en la materia.

Las agencias de calificación, fundadas a inicios del siglo xx en los Estados Unidos de América para la valoración de los títulos que comenzaban a difundirse en el mercado -especialmente de los títulos obligacionales emitidos por las empresas ferroviarias ${ }^{2}-$ son, como es sabido, sujetos privados que se dedican profesionalmente a la redacción de credit reports $^{3}$. Desarrollan en tal modo la función de intermediarios independientes: elaboran y difunden informaciones en el mercado con respecto a la solidez y solvencia de los emisores, así como relativas al riesgo de insolvencia crediticia ligado a específicos instrumentos financieros en

2 El origen del rating puede remontarse al documento History of Railroads and Canals in the United States, publicado por Henry Varnum Poor en $\mathrm{i} 860$, en el cual se trataba de recoger informaciones completas relativas a la situación financiera y operacional de las compañías ferroviarias estadounidenses con miras a favorecer la transparencia financiera de tales empresas a través de la publicación de sus balances. Con referencia a la evolución histórica que ha llevado a las agencias de calificación a tornarse tan indispensables en los mercados mundiales ver, sobre todo, Presti, s.v. "rating", en Enc. dir., Ann. viI, Milano, 2014 , 847 ss.; ID., "Le agenzie di rating: dalla protezione alla regolazione", $7 u s, 2009,68$ ss.; ID., "Take the AAA train: note introduttive sul rating", Analisi giur. econ., 20 I 2, 253 ss.; Di Donna, La responsabilità civile delle agenzie di rating, Padova, 201 2, 25 ss.; Ferri y Lacitignola, Le agenzie di rating, 2. ${ }^{a}$ ed., Bologna, 20 I4, I 7 ss.; Sylla, "A Historical Primer on the Business of Credit Rating", Ratings, Rating Agencies and the Global Financial System, Richard M. Levich, Giovanni Majnoni y Carmen M. Reinhart (eds.), New York, 2002, ig ss.

3 Para la definición de calificación (rating) y de agencias de calificación escogida por el legislador europeo ver el artículo 3 inciso i letras a y b del Reglamento CE n. ${ }^{\circ}$ io6o/2009. En fuerza de tales disposiciones debe entenderse por calificación del crédito "un parecer" relativo al mérito crediticio de una entidad o de sus emisiones, formulado por medio de un sistema de clasificación predeterminado, mientras que por agencia de calificación se entiende "una persona jurídica cuya actividad incluye la emisión de calificaciones del crédito a nivel profesional". Similar es la definición brindada por el IOSCO Code of Conduct Fundamentals for CRAs (disponible en: http:// www.iosco.org/library/pubdocs/pdf/IOsCOPD482.pdf): “Credit rating agency' or 'CRA' means an entity that is in the business of issuing credit ratings" (Appendix A, A-4). 
relación con la situación patrimonial de los otros emisores y emisiones presentes en el mercado. Los resultados de tales operaciones interpretativas se representan mediante símbolos convencionales, elegidos por cada agencia de calificación, que reúnen en un único signo todas las valoraciones, datos y análisis realizados. Así se posibilita en los mercados la difusión de juicios de síntesis simples y de ágil utilización, capaces de ayudar a los inversionistas a tomar óptimas decisiones de inversión. Se atribuye un voto a las emisiones de entidades y de Estados, con el objetivo de ahorrar a los sujetos interesados en una inversión el esfuerzo y los costos de valorar por cuenta propia los riesgos y los posibles rendimientos.

La actividad principal de las agencias de calificación consiste entonces en la recolección de informaciones relativas a los fundamentales económicos y financieros de la entidad evaluada, analizarlas y formular un juicio de carácter pronóstico con respecto a la capacidad, por parte del emisor evaluado, de restituir y remunerar puntualmente todo aquello que fue invertido. Evalúan el riesgo conexo a la inversión en un ente determinando o en un específico instrumento financiero: la "capacidad de crédito" indica, por un lado, la probabilidad de default de un ente o de una emisión, y por otro, su capacidad de resistencia a factores externos de estrés. Por consiguiente, con el término bond rating se entiende la valoración general del riesgo crediticio conexo a una empresa relativo a la totalidad de su exposición de endeudamiento, comprendidas en ella las garantías otorgadas 4 .

La peculiaridad del rol de las agencias de calificación consiste en su posición de intermediarios entre los varios actores en los mercados internacionales. Debido a que las calificaciones son productos "de experiencia", cuya calidad puede ser valorada solamente con el paso del tiempo y con base en confirmaciones factuales sobre la exactitud de las previsiones en ellas formuladas 5 , las agencias pueden elencarse dentro de los llamados reputational intermediaries.

Con tal expresión se hace referencia a sujetos particulares que cumplen una función de mediación entre los actores del mercado, ofreciendo al público de los inversionistas servicios de certificación, análisis y control sobre la situación económica y financiera de los entes evaluados. Su peculiaridad radica en el hecho de que la persuasión, y por tanto el valor de sus declaraciones, deriva de la reputación como expertos que han sabido forjarse con el pasar del tiempo ${ }^{6}$. Tales

4 Por todos, ver Ferri y Lacitignola, Le agenzie di rating, cit., 6i s.; Presti, Le agenzie di rating: dalla protezione alla regolazione, cit., 66; SCARoni, "La responsabilità delle agenzie di rating nei confronti degli investitori", Contratto e impresa, 201 I, 3, 766 ss.

$5 \mathrm{Al}$ respecto ver, sobre el ordenamiento alemán, HAAR, "Das deutsche Ausführungsgesetz zur EU-Rating-Verordnung - Zwischentappe auf dem Weg zu einer europäischen Finanzmarktarchitektur", ZBB, 2009, I 78; Rотт, "Europäisierung des Rechts der Finanzintermediäre", EWS, 2008, 2 I ss.

6 Una contribución esencial en la materia es la de KraAkman, "Gatekeepers: The Anatomy of a Third-Party Enforcement Strategy”, 7. L. Econ. \& Org., I986, 53 ss. Véase además Chor, “Mar- 
sujetos, como lo muestra a primera vista el ejemplo de las agencias de calificación7, funcionan en mercados bastante concentrados y ofrecen sus servicios a un número indeterminado de destinatarios en el plano global. Una agencia, de hecho, puede consolidar su posición en el mercado únicamente a largo plazo, gracias al prestigio obtenido y reforzado en el tiempo, por haber sabido predecir correctamente los riesgos ligados a las entidades y a las inversiones examinadas.

De estas características de las agencias de calificación se pueden extraer dos conclusiones. Ante todo, desde un punto de vista teórico, los mecanismos descritos tendrían que inducir a las agencias a depositar un valor particularmente alto en el mantenimiento de una reputación sólida y ejemplar; y ya esto las debería incitar a difundir en el mercado solamente informaciones correctas y confiables ${ }^{8}$. En segundo lugar, de esta estructura se deriva la actual conformación del mercado de la calificación, que es un mercado a escala mundial connotado por fuertes elementos de oligopolio: se ha mencionado cómo solamente tres agencias de calificación cubren la casi totalidad del mercado.

Ello tiene consecuencias importantes en la operatividad de los mecanismos de competencia que tendrían que maniobrar y dirigir la actividad de las agencias. Las mencionadas peculiaridades del mercado de la calificación han jugado un rol determinante en la distorsión del funcionamiento normal de aquellos automatismos que, según las alegaciones teóricas, habrían tenido que contribuir al buen funcionamiento del mercado de la calificación y, en particular, al control cualitativo de las valoraciones realizadas: en vez de la garantía de una alta calidad de la calificación, fueron en cambio tomando fuerza posiciones dominantes ya presentes independientemente de la exactitud de las opiniones difundidas (e incluso, en ciertos casos, en presencia de gravísimos y comprobados errores de valoración).

ket Lessons for Gatekeepers", Nw. U.L. Rev., 92, I998, 917 ss.; LeYens, "Intermediary Independence: Auditors, Financial Analysts and Rating Agencies", fournal of Corporate Law Studies, 20 I I, 33 ss. En materia de agencias de calificación ver Diтtrich, The Credit Rating Industry. Competition and Regulation, Köln, 2007, 2 I ss.

7 Es bien sabido que solo tres agencias de calificación (Moody's, Standard \& Poor's, Fitch Ratings) cubren una cuota igual al $95 \%$ del mercado internacional de la calificación (en este sentido ver Commissione Europea, Memorando del I6.I.2013, New rules on credit rating agencies (CRAs), I, disponible en: http://europa.eu/rapid/press-release_MEмо-I3-I3_en.htm). Para informaciones más detalladas ver Moody's Annual Report 2014; McGraw-Hill Companies, Annual report 2014. Ambos informes anuales de tales sociedades pueden encontrarse en sus respectivas páginas web. Para Fitch Ratings ver Fimalac, Rapport annuel 20 I4. Sobre el punto ver ESME, Role of Credit Rating Agencies, ESME's report to the European Commission, junio de 2008, i I ss.

8 Para un análisis profundo de estos mecanismos reputacionales ver, en particular, Schwarcz, "Private Ordering of Public Markets: The Rating Agency Paradox", U. Ill. L. Rev. I4, 2002, I ss.; Gilson y Kraakman, "The Mechanisms of Market Efficiency”, Va. L. Rev. 70, I984, 620 ss.; Shaprro, "Premiums for High Quality Products as Returns to Reputations", Quart. F. Econ. 98, I 983,659 ss. En el ordenamiento italiano ver, además, Olivieri, "I servizi di rating tra concorrenza e regolazione", Analisi giur. econ., 201 2, 285 ss.; FACCI, "Le agenzie di rating e la responsabilità per informazioni inesatte", Contratto e impresa, 2008, I, I 73 ss., con ulteriores referencias. 
Las agencias de calificación tienen una enorme influencia en los mercados mundiales: sus juicios representan el criterio principal, a veces incluso el único, que guía las elecciones de inversión y de distribución del ahorro, en beneficio de algunos tomadores más que de otros. Sin embargo, por los motivos que ya se han señalado, tal influencia no se compensa con los normales mecanismos de competencia que tendrían que actuar, debido a que la peculiaridad de las calificaciones, en su calidad de bienes de "experiencia", y las otras barreras fácticas al ingreso de nuevos operadores, impiden que puedan consolidarse nuevos competidores en este mercado fuertemente oligopólico, caracterizado por la presencia de tres grandes agencias 9 . Ello ha ocasionado que las repercusiones esperadas de los mecanismos reputacionales sobre la calidad de las calificaciones no hayan podido manifestarse todavía.

En particular, ha sido puesto de relieve en modo unánime ${ }^{\mathrm{Io}}$ el rol de las agencias de calificación en la generación, así como en la agudización, de la crisis financiera que se produjo entre 2006 y 2007 por los llamados préstamos subprime -o sea, aquellos préstamos concedidos a una clientela caracterizada por un credit score particularmente bajo, o financiamientos muy amplios concedidos a deudores que de otro modo no habrían tenido un buen mérito de crédito $^{\mathrm{II}}$. Las agencias actuaron en franco conflicto de intereses, valorando instrumentos financieros que ellas mismas habían contribuido a estructurar por auxiliary services antecedentes, e hicieron uso, sin la necesaria corrección y transparencia, de

9 Sobre el tema ver, ante todo, Pinto, "Control and Responsibility of Credit Rating Agencies in the United States", Am. F. Comp. L., 54, 2006, 343 ss., donde se encuentran ulteriores referencias.

Io Ver The Financial Crisis Inquiry Report, National Commission on the Causes of the Financial and Economic Crisis in the United States, $20 \mathrm{I}$ I, xxv, 43 ss. (disponible en: https:/www.gpo.gov/fdsys/pkg/ GPO-FCIC/pdf/GPO-FCIC.pdf). Ver, además, el informe contenido en la propuesta de Reglamento сом (2008) 704 del I 2 de noviembre 2008, 2. En el panorama doctrinario internacional ver, en particular, Avgouleas, "The Global Financial Crisis - Behavioural Finance and Financial Regulation - In Search of a New Orthodoxy", 7. Corp. L. Studies 9, 2009, 33 ss.; Partnoy, "Overdependence on Credit Rating Was a Primary Cause of the Crisis", San Diego Legal Studies o9/o I , 2009, 5 ss.; Turner, The Turner Review 2009 - A Regulatory response to the Global Financial Crisis, (disponible en: http://www.fsa.gov.uk/pubs/other/turner_review.pdf); BAR-GILL, "The Law, Economics and Psychology of Subprime Mortgage Contracts", Cornell L. rev. 94, 2009, I073 ss.; Grundmann, Hofmann y Möslein, "Finanzkrise und Wirtschaftsordnung: Krisenursachen, Finanzmarktstabilisierung, Finanzmarktstabilität", Finanzkrise und Wirtschaftsordnung al cuidado de Grundmann, Hofmann y Möslein, Berlin, 2009, I, 3 ss.; Wagner, "Die Haftung von Ratingagenturen gegenüber dem Anlegerpublikum", Einheit und Vielheit im Unternebmensrecht, Festschrift für Uwe Blaurock zum 7o. Geburtstag, Jung, LAmprecht et al. (eds.), Tübingen, 20I3, 469 ss.; KumPan, "Regulierung von Ratingagenturen - ein anreizorientierter Ansatz", Festschrift für Klaus 7. Hopt zum 7o. Geburtstag, vol. II, Grundmann et al. (eds.), Berlin, 2010, 2 I 57 ss.; Presti, "Le agenzie di rating: dalla protezione alla regolazione", fus, 2009, 78 ss.; Facci, "Le agenzie di rating e la responsabilità per informazioni inesatte", Contr. e impr., 2008, I64 ss. tdown”, Minnesota Law Review, 93, 2008, 373, nota I. 
criterios de valoración inidóneos y de datos obtenidos por los entes evaluados sin control de fondo alguno.

Con todo y ello, estos comportamientos no han implicado consecuencia negativa alguna para las agencias en mención.

Lo ocurrido se ubica en el vértice de una escalada de grandes escándalos financieros, como aquellos de Enron de $200 \mathrm{I}^{\mathrm{I2}}$, WorldCom de $2002^{\left[{ }^{[} 3\right]}$ y Parmalat de 2003 , en los que fueron demandadas también las agencias de calificación ${ }^{\mathrm{I}}$. Luego de tales eventos resultó fuertemente golpeado el rol de las agencias como gatekeepers $^{\mathrm{I} 5}$ eficientes, debido a que en todas estas circunstancias no pudieron (o tal vez no quisieron) advertir al mercado sobre las dificultades financieras de las sociedades evaluadas, manteniendo alta su calificación hasta pocos días antes de la declaración de quiebra ${ }^{\mathrm{I} 6}$.

La reacción global a tales comportamientos inicialmente se limitó al soft law. La organización internacional de las autoridades de vigilancia sobre los mercados financieros (IOsCO) se manifestó publicando un reporte ${ }^{17}$ y una declaración sobre los principios a seguir en la actividad de calificación ${ }^{18}$, que dieron lugar sucesivamente al Iosco Code of Conduct Fundamentals for CRAs, de $2004^{\text {[19]. }}$ En una materia dejada hasta aquel entonces a la total autonomía de los sujetos implicados, se introdujo así un primer núcleo de principios y de reglas de comportamiento para las agencias de calificación, entendidos como indicaciones y

I 2 Ver Financial Oversight of Enron: The SEC and Private-Sector Watchdogs, Report to the Staff to the Senate Committee on Governmental Affairs, US Senate 7 de octubre de 2002 (disponible en: https://www.gpo.gov/fdsys/pkg/CPRT-IO7SPRT82 I47/pdf/CPRT-IO7SPRT82 I47.pdf). Sobre el tema ver, también, Armour y McCahery, "After Enron: Improving Corporate Law and Modernizing Securities Regulation in Europe and in the US", Amsterdam Center for Law \& Economics Working Paper No. 2006-o7, I ss.

I 3 Al respecto, ver Coffee, Gatekeepers. The Professions and Corporate Governance, Oxford, 2006, 36 ss.

I4 Los acontencimientos son bien conocidos. Sobre el tema ver las observaciones de BuOnOcore, "Etica degli affari e impresa etica", Giur. comm., 2004, I8 I ss.

I 5 Para una visión panorámica sobre la primera literatura estadounidense en tema de gatekeepers ver Kraakman, "Corporate Liability Strategies and the Costs at Legal Controls", Yale L. F., 93, I984, 857 ss.; ID., "Gatekeepers: The Anatomy of a Third-Party Enforcement Strategy", 7. L. Econ. \& Org., 2, I986, 53 ss.; GiLson, "Value Creation by Business Lawyers: Legal Skills and Asset Pricing", Yale L. 7., 94, I 984, 239 ss.; Chor, "Market Lessons for Gatekeepers", Nw. U.L. Rev., 92, I998, 916 ss.

I6 $\mathrm{Al}$ respecto ver, sobre todo, Presti, Le agenzie di rating, cit., 78 ss.; Drigo, "La responsabilità delle agenzie di rating per il danno all'informato. L'esperienza statunitense", Rass. dir. civ., 2006, 490 ss.

I 7 Iosco, Report on the Activities of Credit Rating Agencies (disponible en: www.iosco.org).

I 8 Iosco, Statement of Principles Regarding the Activities of Credit Rating Agencies de 2003 (disponible en: www.iosco.org).

I9 Disponible en línea, en su versión actualizada al año 2005, en: http://www.iosco.org/library/ pubdocs/pdf/Ioscopd482.pdf 
guías sobre cómo estructurar los respectivos códigos de autorregulación ${ }^{20}$. Este primer intento de intervención, tomado por las calificadoras en forma de adhesión voluntaria y carente de un efectivo poder impositivo, se reveló a la luz de los hechos como insuficiente, por lo inadecuado para poder enfrentar la crisis de los mutuos subprime. Así las cosas, hubo de pasarse de la autorregulación a la heterorregulación ${ }^{21}$.

El legislador europeo, inicialmente sordo a las preocupaciones y a las instancias de tutela ya formuladas por parte de la doctrina más atenta ${ }^{22}$, solo entró en acción con la crisis que afectó las finanzas mundiales a partir del año 2006. Las consiguientes reflexiones de política del derecho se desarrollaron paralelamente con aquellas llevadas a cabo al otro lado del océano, en los Estados Unidos de América $^{23}$, y ello trajo como consecuencia una serie de intervenciones normativas dirigidas a regular el comportamiento de las agencias de calificación: el Reglamento CE n. ${ }^{\circ}$ Io6o/2009, del r6 de septiembre de 2009, sobre las agencias de calificación, y dos reglamentos modificatorios sucesivos, a saber, el Reglamento UE n. ${ }^{\circ}$ 5I3/20I I, del I I de mayo de 20I I, y el Reglamento UE n. ${ }^{\circ}$ 462/20I3, del 2 I de mayo de 2013 .

Sobre el tema ver Presti, Le agenzie di rating, cit., 79 ss.

2 I Sobre este cambio de visión ver Cazorla Prieto, "La inserción de las agencias de 'rating' en el incipiente gobierno de la globalización financiera", Derecho Bancario y Bursátil, n. ${ }^{\circ}$ I 29, 2013 , $2 \mathrm{I} 3$

22 La Comisión Europea, de hecho, estuvo en contra de una intervención regulatoria en esta materia en el año 2005 (ver Comunicazione della Commissione europea sulle agenzie di rating del credito del 23 de diciembre de 2005, en Gazzetta ufficiale dell'UE 2006/C 59/o2 del I I de marzo de 2006, espec. 9 s.). Del mismo modo, el Grupo de Expertos de los Mercados Europeos de los Valores Mobiliarios (European Securities Markets Expert Group, ESME), incluso ya en el año 2008 (ESME, Role of Credit Rating Agencies, en ESME's Report to the European Commission, junio de 2008, 9 s.). Sobre el punto ver, en particular, Carriero, "Previsioni, opinioni, certezze: le agenzie di rating”, Europa e dir. priv., 2OI2, 874; DrIGO, La responsabilità delle agenzie di rating, cit., $492 \mathrm{~s}$. y 499 s. En ámbito alemán ver Schuler, Regulierung und zivilrechtliche Verantwortlichkeit von Ratingagenturen, Frankfurt a.M., 201 2, 94 ss.

23 El Credit Rating Agency Reform Act of 2006, emanado para enfrentar la situación crítica de las agencias de calificación, puesta de presente por el default de Enron y el de WordCom, inmediatamente resultó inadecuado para afrontar la crisis de los mutuos subprime (ver HunT, "Credit Rating Agencies and the 'Worldwide Credit Crisis': The Limits of Reputation, The Insufficiency of Reform and a Proposal for Improvement", Colum. Bus. L. Rev. Io9, 2009, 23 s.). La crisis representó un verdadero punto de inflexión tanto en la evolución de la jurisprudencia en materia de responsabilidad de las agencias de calificación como en el desarrollo de la legislación. Punto de llegada de tal progreso es la emanación del Dodd-Frank Wall Street Reform and Consumer Protection Act (Pub. L. I I I-203, H.R. 4I 73), firmado por el presidente Obama el 2 I de julio de 20IO. Al respecto ver, sobre todo, Hill, "Limits of Dodd-Frank's Rating Agency Reform", Chapman L. Rev., I 5, 20 I I, I43 ss. (y en Le agenzie di rating, Principe (ed.), Milano, 20I4, I2 ss.). 


\section{La introducción de una reglamentación europea en materia de calificación}

Ya en el año 2003 el Parlamento europeo había encargado a la Comisión el hacer una reflexión sobre la necesidad de adoptar disposiciones normativas específicas en materia de calificación ${ }^{24}$. A raíz de esta decisión, la Comisión requirió soporte técnico al Committee of European Securities Regulators (CESR) para iniciar los trabajos de redacción de un texto normativo adecuado ${ }^{25}$. Se empezaba a sentir preocupación por la presencia de un vacío legislativo en un ámbito tan relevante para la economía europea, y al mismo tiempo dejó de considerarse como suficiente dejar que la reglamentación del fenómeno de la calificación recayera exclusivamente en el derecho nacional de los Estados miembros.

La Comisión, sin embargo, no tuvo la misma opinión que el Parlamento y afirmó, en una comunicación del año 2005 , que no consideraba necesaria una nueva iniciativa legislativa en la materia ${ }^{26}$. Por un lado, ponía el acento en la reglamentación europea existente que ya tocaba en parte la actividad de las agencias de calificación, disciplinando los aspectos que se consideraban como los más problemáticos: por ejemplo, en materia de abuso de informaciones privilegiadas y de manipulación del mercado ${ }^{27}$, o en materia de utilización, por parte de los bancos, de evaluaciones del mérito del crédito externas a la determinación de las ponderaciones de riesgo ${ }^{28}$. Por otro lado, consideraba que podía ver, en este ámbito, un adecuado equilibrio entre las intervenciones legislativas y la autorregulación.

Tras los terremotos financieros que alteraron el panorama internacional, finalmente se afirmó al interior de la Comisión europea la convicción de la necesidad de delinear una disciplina común a todos los Estados miembros de la Unión Europea, y aquella de la insuficiencia de las reglas europeas ya existentes sobre la actividad de la calificación. Se optó entonces por la codificación de claros deberes de comportamiento de las agencias de calificación, y posteriormente, por la creación de una base normativa común sobre la responsabilidad de las agencias mismas. Estas reflexiones trascendieron en el Reglamento CE n. ${ }^{\circ}$ io6o/2009 del

24 Resolución del Parlamento europeo sobre el rol y los métodos de las agencias de calificación 2003/208 I (INI).

25 CEsR, Technical advice to the European Commission on Possible Measures Concerning Credit Rating Agencies, con fecha 30 de marzo de 2005.

26 Ver Comunicación de la Comisión sobre las agencias de calificación del crédito 2006/C 59/02, cit., 9 s. En el mismo sentido ver Esme, Role of Credit Rating Agencies, EsME's Report to the European Commission, junio, 2008, 22 ss. Sobre el punto, en detalle, Carriero, Previsioni, opinioni, certezze: le agenzie di rating, cit., 874 .

27 La referencia es a la Directiva CE n. ${ }^{\circ}$ 6/2003, relativa al abuso de informaciones privilegiadas y a la manipulación del mercado.

28 Ver Directiva CE n. ${ }^{\circ} 48 / 2006$, relativa al acceso a la actividad de los entes crediticios y a su ejercicio. 
Parlamento europeo y del Consejo, de i6 de septiembre de 2009, referido a las agencias calificadoras del crédito, y en sus sucesivos reglamentos de modificación.

El complejo normativo tiene un ámbito de aplicación bien definido. Disciplina únicamente las calificaciones que satisfagan dos requisitos esenciales, enunciados por el artículo 2 inciso I del Reglamento recientemente citado: in primis deben haber sido emitidas por agencias especializadas que operen en el territorio europeo y que estén registradas en la Comunidad como "ECAI" con arreglo a la Directiva CE n. ${ }^{\circ} 48 / 2006^{[29]}$; in secundis deben ser "comunicadas al público o distribuidas previa suscripción”. Así, se excluyen del ámbito de aplicación del Reglamento todas las calificaciones no emitidas por $\mathrm{ECAI}^{30}$, ya se trate de calificaciones privadas no destinadas a ser divulgadas, puntajes sobre la confiabilidad crediticia de los credit scores, calificaciones producidas para el crédito a las exportaciones, y algunas tipologías de conceptos formulados por los bancos centrales de los Estados miembros ${ }^{3}$.

De este modo, se quiso circunscribir la disciplina a los supuestos más relevantes, también en un plano cuantitativo, en los cuales la necesidad de intervención era más urgente debido a que se comprometían o resultaban amenazados los mercados financieros. Se reconoció así que en ciertos ámbitos, en los que la influencia de las agencias y la función de los gatekeeping es mayor en un plano reglamentario, los peligros de disfunción para el sistema financiero son bastante marcados, mientras que en los otros casos, en los que la actividad de clasificación, por su naturaleza, no está destinada a su divulgación y más bien lo está a manifestar sus efectos en un restringido ámbito personal, no se presentan problemas

29 Una agencia de calificación puede ser reconocida como agencia externa de valoración del mérito de crédito (o External Credit Assessment Institution, de ahí el acrónimo ECAI) si cumple con los requisitos fijados en la parte 2 del Anexo vi de la Directiva CE n. ${ }^{\circ} 48 / 2006$, relativos tanto a la metodología utilizada para la definición de los juicios de calificación (y por lo tanto a su objetividad, independencia, revisión periódica, transparencia) como a las valoraciones que de allí derivan (que deben ser creíbles y transparentes). La verificación de los requisitos y la reconducción de las calificaciones a clases de ponderación ('mapping') son efectuados por el Banco de Italia sobre la base de los criterios previstos por la sección viII de la Circular n. ${ }_{2} 63$ del 27 de diciembre de 2006. Las EcAI reconocidas por las autoridades de vigilancia de otro Estado comunitario pueden ser reconocidas por el Banco de Italia sobre la base de la evaluación efectuada por las autoridades de vigilancia en cuestión. En este caso se habla de reconocimiento indirecto.

30 A la lista completa de las EcAI reconocidas en Europa por la European Banking Authority en colaboración con la European Securities and Markets Authority (Autoridad Europea de los Instrumentos Financieros y del Mercado) y la European Insurance and Occupational Pensions Authority (EIOPA) puede tenerse acceso en línea en la página web de la EBA: http://www.eba.europa.eu/regulation-and-policy/external-credit-assessment-institutions-ecai En ella se encuentran las agencias de calificación más importantes que operan en el ámbito europeo.

Ver art. 2 inc. 2 Reglamento CE n. ${ }^{\circ}$ Io60/2009. 
de tipo regulatorio, y por lo tanto no es necesaria una intervención normativa por parte de la Unión ${ }^{2}$.

Así las cosas, en los supuestos comprendidos en el ámbito de aplicación del artículo 2 del Reglamento CE n. ${ }^{\circ}$ ıo6o/2009, el legislador europeo identificó precisos deberes de comportamiento que las agencias de calificación están obligadas a respetar. A una primera formulación de los deberes de comportamiento, contenida en la versión originaria del citado Reglamento, se agregó luego una especificación detallada por cuenta del primer reglamento modificatorio, esto es, el Reglamento UE n. ${ }^{\circ}$ 5 I3/20 I I, el cual agregó un Anexo III al Reglamento sobre la calificación, a su vez modificado por el Reglamento UE n. ${ }^{\circ}$ 462/20I3. Se delinea así un cuadro detallado de las conductas que deben ser respetadas por las agencias de calificación; entre estas, en particular, el deber de independencia frente a las entidades evaluadas y frente a cualquier otro poder político o económico ${ }^{33}$, el deber de transparencia ${ }^{34}$, el deber de predisposición de sistemas organizativos, administrativos, de control y de contabilidad adecuados ${ }^{35}$, y el deber de diligencia profesional ${ }^{36}$.

Los deberes de comportamiento delineados tienen como fin, en su conjunto, garantizar una alta calidad de los juicios de calificación y del procedimiento que se sigue para obtenerlos. En este sentido, corresponden en gran parte a las disposiciones materiales contenidas en las recomendaciones de la Iosco, en donde se plantean las problemáticas relativas a la necesaria objetividad y neutralidad de las agencias de calificación, la competencia de los sujetos que realizan las evaluaciones y la importancia para las agencias de disponer de recursos humanos

32 En este sentido ver también Parmeggiani, "La regolazione delle agenzie di rating tra tentativi incompiuti e prospettive future", Giur. comm., 2010, I, I 35 s.

$33 \mathrm{Al}$ respecto de este deber de comportamiento ver el artículo 6 inciso I, según el cual las agencias de calificación del crédito están obligadas a adoptar las medidas necesarias para asegurar "que la emisión de una calificación no esté influencia por algún conflicto de interés, existente o potencial, o por relaciones de negocios relativas a la agencia emisora de la calificación, a sus directores, analistas de calificación, dependientes o a cualquier otra persona física"; el artículo 6 ter prevé un deber de rotación para la evaluación del mérito del crédito para las operaciones de retitularización de los créditos; el artículo 7 prevé en el inciso 2 que las personas físicas que participen directamente en la actividad de calificación del crédito no están autorizadas ni a iniciar, ni a participar en negociaciones relacionadas con la compensación por la actividad desarrollada; y en el inciso 4 se introduce un sistema de rotación gradual de los analistas y de los otros sujetos comprometidos en la emisión de la calificación. Ver además el Anexo i, secc. A, inc. I y el Anexo iII, secc. I puntos 4 ss. Ver López Martínez, "Independencia y conflictos de interés en la actividad de las agencias de rating”, Rev. derecho bancario y bursátil, n. ${ }^{\circ}$ I 29, 20 I $_{3}, 238$ ss., quien subraya la importancia fundamental de este deber y su relieve sistemático.

34 Ver Considerandos 25 ss. del Reglamento CE n. ${ }^{\circ}$ Io6o/2009, el art. 6 inc. 2 , los arts. 8 a I I, el Anexo I, sección B.

35 Considerandos 28 ss. del Reglamento CE n. ${ }^{\circ}$ Io6o/2009, art. 6 inc. 4, Anexo I, sección A y B.

36 Considerandos 34 y 35 del Reglamento CE n. ${ }^{\circ}$ Io6o/2009, art. 8 inc. 2 ss., Anexo I, secc. D, Anexo III, secc. I n. ${ }^{\circ} 2$ ss. 
suficientes ${ }^{37}$. Se trata, por lo demás, de un objetivo que la Comisión misma ya había considerado, al considerar el Code of Conduct Fundamentals como un "parámetro de referencia mundial", que carece, sin embargo, de concreción y consolidación, y sobre todo de un sistema coercitivo eficaz ${ }^{3}$.

El Reglamento CE n. ${ }^{\circ}$ Io60/2009 se encuadra entonces en un plano completamente diferente en relación con estas normas: atribuye un nuevo valor jurídico a los estándares de organización internacional relativos al grado de diligencia que se puede y se debe requerir a las agencias de calificación en el desarrollo de su actividad. Las reglas dejan de ser sugerencias o indicaciones a las agencias, y de estar destinadas a su voluntaria adhesión en los respectivos códigos de conducta, para convertirse en disposiciones jurídicamente vinculantes del legislador europeo, obligatorias en todos sus elementos y aplicables directamente en todos los Estados miembros, tanto en las relaciones verticales como horizontales, sin necesidad de intervenciones ulteriores de recepción por parte de los legisladores nacionales.

La violación de los mencionados deberes de comportamiento asume en el sistema de los reglamentos europeos en materia de calificación una doble relevancia. Bajo un primer aspecto, conduce a la imposición por parte de las autoridades dotadas de poderes de vigilancia (ESMA) de sanciones de naturaleza tanto administrativa como penal, de conformidad con los artículos 24 y 36 del Reglamento CE n. ${ }^{\circ}$ Io60/2009 y sus modificaciones sucesivas ${ }^{39}$. Por otra parte, emerge con claridad cómo los deberes de comportamiento descritos aportan elementos centrales para la determinación del nivel de diligencia al que deben atenerse las agencias de calificación, y por ello tienen un impacto definitivo en la responsabilidad de las agencias de calificación ${ }^{4}$. Precisamente a ellos se vincula un remedio resarcitorio en protección de los inversionistas y de los emisores lesionados, introducido más recientemente por el Reglamento UE n. ${ }^{\circ}$ 462/2013. Con el segundo reglamento modificatorio se introdujo en el Reglamento CE n. ${ }^{\circ}$

Ver, respectivamente, los puntos n. ${ }^{\circ}$ I.2-I.7, 2.I-2.I 7 y I.7-I.8 del Iosco Code of Conduct Fundamentals.

38 Ver el informe a la propuesta de reglamento del Parlamento europeo y del Consejo del I 2 de niviembre de 2008, ком (2008) 707, 3 .

39 La Autoridad Europea de los Instrumentos y de los Mercados (en inglés, European Securities and Markets Authority, ESMA), constituida con posterioridad al Reglamento UE n. ${ }^{\circ}$ I095/2010, que instituye la autoridad europea de vigilancia, fue individuada como el órgano competente de vigilancia de la actividad de las agencias de calificación en virtud del primer reglamento de modificación del Reglamento sobre la calificación, es decir, el Reglamento UE n. ${ }^{\circ}$ I $3 / 20$ I . $^{2}$ Sobre la materia ver TÁpia Hermida, "Novedades en la regulación comunitaria y española de las agencias de calificación crediticias (agencias de rating)", Derecho Bancario y Bursátil, n. ${ }^{\circ}$ I 23 , 20 I I 296 ss.; García Alcubilla, "La supervisión de las agencias de rating en Europa", Derecho Bancario y Bursátil, $\mathrm{n}^{\circ}{ }^{\mathrm{I}}$ 2 29, 2013 , 2 I 9 ss. Antes de tal intervención, la vigilancia relativa a la aplicación del Reglamento, junto con la determinación e imposición de las sanciones, era solicitada a los Estados miembros (ver art. 36 Reglamento CE n. ${ }^{\circ}$ io6o/2009 en su versión original).

Ver en este sentido ya el informe del Reglamento CE n. ${ }^{\circ}$ Io60/2009, en particular pars. 23 y 25 . 
I060/2009 el artículo 35 bis, que sanciona la responsabilidad de las agencias de calificación frente a los acreedores y emisores cuando hayan violado con dolo o con culpa grave los deberes de comportamiento descritos en el Anexo III del Reglamento mismo ${ }^{4}$.

\section{El segundo reglamento modificatorio del Reglamento CE n. ${ }^{\circ}$ I $060 / 2009$}

En un contexto como el que es objeto de examen, es imposible no notar cómo la responsabilidad civil de las agencias de calificación presenta un significado particular. En efecto, ella no asume un rol únicamente como instrumento de compensación, puesto a disposición de los actores del mercado que resultan injustamente lesionados por la publicación de una calificación, sino que más en general, desde un punto de vista macroeconómico, se quiso introducir un mecanismo de control capaz de fungir como instrumento disuasivo de comportamientos incorrectos por parte de las agencias, para incentivar el buen funcionamiento del mercado de calificación y la praxis de evaluación más precisa y confiable posible ${ }^{42}$. A estos dos fines aspira la codificación de una responsabilidad en caso de violación de deberes normativos específicamente codificados, concretada en el segundo reglamento de modificación al Reglamento europeo en materia de calificación 43 .

Como consecuencia del fracaso del modelo reputacional, centrado en las sanciones de mercado, pareció indispensable introducir una sanción jurídica capaz de desincentivar eficazmente los comportamientos oportunistas de las agencias -las cuales, como es sabido, no actúan movidas por fines de solidaridad ni de utilidad social- enfocados en la maximización de la propia utilidad, en detrimento de la generalidad de los otros operadores del mercado. Así entonces se delineó

4I Tal anexo se introdujo por el primer reglamento modificatorio (n. ${ }^{\circ}$ I $3 / 20$ I $^{\text {I }}$ y fue integrado sucesivamente al segundo reglamento modificatorio (n. ${ }^{\circ} 462 / 2013$ ).

42 En este sentido ver, para el ordenamiento italiano, Cervone, "Riflessioni per un'indagine sulle agenzie di rating", Riv. trim. dir econ., 2009, I, 293 ss.; Perrone, Informazione sul mercato e tutele dell'investitore, Milano, 2003, 38 ss. En la literatura estadounidense ver además Crawford, "Hitting the Sweet Spot by Accident: How Recent Lower Court Cases Help Realign Incentives in the Credit Rating Industry", Conn. Law Rev., 42, 2009, i 8; Husisian, "What Standard of Care Should Govern the World's Shortest Editorials?: An Analysis of Bond Rating Agency Liability", Cornell L. Rev., 75, 4Io, I990, 430; Pinto, "Control and Responsibility of Credit Rating Agencies in the United States", Am.F. Comp. L., 54, 2006, 35 I; Rousseau, "Enhancing the Accountability of Credit Rating Agencies: The Case for a Disclosure-Based Approach”, McGill L.7., 5 I, 2006, 642. En el ordenamiento alemán ver además Schroeter, Ratings, cit., 778 s.; Blaurock, "Verantwortlichkeit von Ratingagenturen - Steuerung durch Privat- oder Aufsichtsrecht?", ZGR, 2007, 642; Habersack, "Rechtsfragen des Emittenten-Ratings", ZHR, I69, 2005, I89.

43 Con referencia a la solución llamada second best, desde un punto de vista teórico, ver particularmente Lipsey y Lancaster, "General Theory of Second Best", Rev. Econ. Stud. 24, I956, i I ss. 
un sistema de responsabilidad civil dirigido a incentivar un ejercicio correcto de la actividad de las agencias.

Se ha puesto de relieve que para los inversionistas y para los otros sujetos del mercado la perspectiva de obtener una posible ventaja económica bajo la forma de resarcimiento del daño padecido constituye un estímulo importante para la búsqueda e individuación de comportamientos incorrectos por parte de las agencias de calificación 44 . Y no se puede ignorar que la amenaza de una posible responsabilidad civil es capaz, de facto, de cumplir una función preventivodisuasiva ${ }^{45}$. Así viene a materializarse la capacidad de la responsabilidad civil de enderezar el comportamiento de las agencias de calificación ${ }^{4}$.

Es también en consideración a las funciones fundamentales propias de la responsabilidad civil, en una materia tan sensible y relevante para los mercados internacionales, que la intención inicial del legislador europeo de delegar in toto la cuestión al derecho nacional47 fue sustituida en un segundo momento por la voluntad de intervenir directamente una temática tan delicada. De hecho, de estas reflexiones nació la base jurídica autónoma de la pretensión de derecho europeo al resarcimiento del daño de la cual se ocupa el artículo 35 bis del Reglamento CE n. ${ }^{\circ}$ io6o/2009 ${ }^{[48]}$.

44 Ver, por todos, HaAR, "Die Rolle der Ratingagenturen bei der Corporate Governance von Banken", Handbuch Corporate Governance von Banken, Hopt y Wohlmannstetter (eds.), München, 20 I I, 233 ss.

45 Entre otros ver, en particular, Montalenti, "Le agenzie di rating: appunti", Giur. comm., 201 3, I, 5 I 4 s.; Cervone, Riflessioni per un'indagine sulle agenzie di rating, cit., 293 ss.; Perrone, Informazione sul mercato e tutele dell'investitore, cit., 38 ss. En el ordenamiento alemán véanse además las agudas reflexiones de SCHÄFER, "Strafe und Prävention im Bürgerlichen Recht", en $A c P, 202$, 2002, 399 ss.; WAGNER, "Prävention und Verhaltenssteuerung durch Privatrecht - Anmaßung oder legitime Aufgabe?", AcP, 206, 2006, 363 s.; HABERSACK, Rechtsfragen des Emittenten-Ratings, cit., I9O s. Entre los autores norteamericanos ver Darbellay y Partnoy, "Credit Rating Agencies and Regulatory Reform", Research Handbook on the Economics of Corporate Law, ClaIRe A. Hill y Brett H. McDonnell (eds.), Cheltenham (UK) - Northhampton (USA), 20I 2, $290 \mathrm{~s}$.

46 Con referencia a la capacidad de la responsabilidad civil de enderezar los comportamientos de los sujetos implicados ver, en particular, el fundamental trabajo de Calabresi, The Costs of Accidents, A Legal and Economic Analysis, New Haven, I970, 26 ss.

47 En este sentido ver el Considerando 69 del Reglamento CE n. ${ }^{\circ}$ Io60/2009: "eventuales peticiones de resarcimiento en contra de las agencias de calificación del crédito por violación de las disposiciones del presente Reglamento tendrían que ser propuestas por norma de la legislación nacional pertinente en materia de responsabilidad civil".

48 Para un análisis del complejo procedimiento legislativo que condujo a tales resultados ver DI Donna, La responsabilità civile delle agenzie di rating, cit., , 08 ss. Los motivos para este cambio de perspectiva pueden atribuirse a dos circunstancias. Por un lado, es relevante el endurecimiento de la responsabilidad de las Credit Rating Agencies en los Estados Unidos de América por cuenta del Dodd-Frank-Act. Sin embargo, fue decisivo que, en el ámbito de la crisis por endeudamiento de algunos Estados soberanos miembros de la Unión Europea, la actividad de las agencias de calificación volvió nuevamente al centro de la atención pública (cfr. Considerando I Reglamento UE n. $\left.{ }^{\circ} 462 / 2013\right)$. A estas se reprochó nuevamente el haber tenido una influencia determinante en la asunción de determinadas decisiones políticas y en el retrasar la superación de la crisis. Sobre el tema ver, en la doctrina italiana, Carriero, Previsioni, opinioni, certezze: le 
El legislador europeo, consciente de la gran importancia de las calificaciones de crédito para las decisiones de los inversionistas, así como de las repercusiones que pueden tener en la imagen y en el atractivo financiero de los emisores, llegó a reconocer las graves dificultades con que los inversionistas y los emisores se encuentran al momento de solicitar, con base en el derecho nacional aplicable, su derecho al resarcimiento del daño ocasionado por la difusión en el mercado de juicios de calificación inexactos formulados por una agencia de calificación, especialmente en caso de ausencia de una relación contractual entre el causante y la víctima del daño49.

\section{Los presupuestos para una responsabilidad de las agencias de calificación frente a emisores e inversionistas}

El Reglamento europeo en materia de calificación contiene a partir del año 2013 una responsabilidad específica por las consecuencias dañinas ocasionadas a emisores e inversionistas como consecuencia de la difusión de evaluaciones inexactas en el mercado; el supuesto de hecho que genera la responsabilidad está atado a la violación de las otras previsiones establecidas por el Reglamento europeo de vigilancia sobre las agencias, cuando de tal conducta violatoria resulte un daño a los inversionistas o a los emisores.

Luego de esta primera y relevante afirmación de principio, el contenido dispositivo del artículo 35 bis es escaso y remite, para muchos aspectos no tratados, y sobre todo para la interpretación y aplicación de los términos que no encuentran una definición específica en el Reglamento, al derecho nacional aplicable al caso concreto. La determinación de conceptos de relevancia central en el ámbito de la cuestión de la responsabilidad, como emerge claramente del elenco ejemplificativo presentado por el legislador mismo, es delegada in toto a los Estados miembros: 'daño', 'intención', 'culpa grave', 'confianza razonable', 'debida atención', 'incidencia', 'razonabilidad', 'proporcionalidad' no son llamados a ser interpretados de manera uniforme según los cánones del derecho europeo, sino sobre la base del derecho nacional de que se trate ${ }^{\circ}$.

Por otra parte, la previsión de una responsabilidad resarcitoria específica de las agencias de calificación, como lo establece expresamente el inciso 5, no excluye el ejercicio de ulteriores acciones de reparación de conformidad con el derecho nacional.

agenzie di rating, cit., 873 ss. En la doctrina alemana ver, además, las interesantes observaciones de HaAr, "Neues zur Haftung von Ratingagenturen im Zuge der zweiten Novelle der RatingVerordnung (CRA III)?”, DB, 2013 3, 2492 s.; WAGNER, Die Haftung von Ratingagenturen gegenüber dem Anlegerpublikum, cit., 487 ss. 
Se encuentran disciplinadas las pretensiones de reparación tanto de los emisores como de los inversionistas. En cuanto a los primeros, se prevé, por una parte, que están legitimados para hacer valer la responsabilidad de las agencias únicamente aquellos emisores que resulten afectados por una calificación injustificadamente baja que provenga de aquellas, o por haber sido objeto de la valoración en la propia entidad o con relación a sus propias emisiones, y por otra, que la violación de los deberes de comportamiento endilgada a las agencias no haya sido producto de informaciones erradas o engañosas entregadas por ellos mismos a la agencia. A su vez, los inversionistas lesionados tienen que estar en la capacidad de demostrar que decidieron adquirir, conservar o ceder un instrumento financiero por haber confiado, a pesar de haber empleado la debida diligencia, en una calificación de crédito equivocada. Si además se trata de inversionistas institucionales, según el artículo 5 bis inciso I del Reglamento, están obligados a hacer una propia valoración del riesgo del crédito sin confiar de manera exclusiva o automática en las valoraciones efectuadas por las agencias.

Con referencia a las pretensiones resarcitorias de los inversionistas -sean ellos o no instituciones- resulta particularmente relevante la precisión de que también la decisión de conservar y no vender un instrumento financiero como consecuencia de una calificación excesivamente positiva puede fundamentar una pretensión de indemnización por parte del inversionista. No ha de olvidarse que también de estos eventos pueden generarse daños significativos a los inversionistas en caso de una inesperada insolvencia del emisor, como lo demuestran los casos Enron y Lebmann Brothers. Pero también la venta de instrumentos financieros puede terminar acarreando un daño a los inversionistas: cuando ella tiene lugar por una calificación excesivamente baja, en la cual confíe el inversionista, la venta se realiza sin lugar a dudas por un valor inferior a aquel que el título habría tenido en el mercado si la calificación hubiese sido la correcta.

El supuesto de responsabilidad previsto en el artículo 35 bis del Reglamento comprende, sin hacer distinción alguna, casos en los que se puede reconocer en las relaciones entre víctima y causante del daño un vínculo obligacional piénsese, ej., en la responsabilidad de las agencias de calificación de frente a los emisores que les hayan encargado una evaluación de bondad crediticia- y casos en los que una relación similar no se presenta ${ }^{5}$. La misma disciplina se aplica cuando quienes solicitan el resarcimiento del daño son emisores evaluados como unsolicited o solicited ${ }^{2}$, o inversionistas que hayan confiado en una calificación errada. Sin embargo, es evidente que la diversa configuración de los diferentes supuestos de responsabilidad tiene una influencia determinante en la conformación efectiva de la pretensión de resarcimiento que la víctima puede hacer valer,

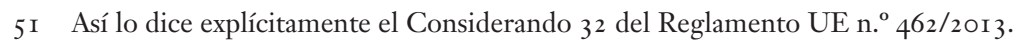

52 Ver Muñoz García, "Los rating no solicitados”, Derecho Bancario y Bursátil, n. ${ }^{\circ}$ I 29, 20 I 3,246 ss. 
in primis en lo que respecta a la cualificación misma de la pretensión y, como consecuencia, en la disciplina de derecho nacional aplicable en la integración de las lagunas dejadas por el derecho europeo.

En cuanto al elemento subjetivo del supuesto, se limita por el artículo 35 bis del Reglamento al dolo o a la culpa grave. La adecuación de tal parámetro de culpabilidad es justificada por el legislador europeo en consideración a la complejidad del procedimiento de calificación, ya que debe considerar variados factores económicos y puede ser desarrollado atendiendo a metodologías diversas, de modo que puede llegarse a juicios discordantes, pero igualmente fundamentados. Solamente en presencia de una culpa particularmente evidente, estima el legislador, se justifica exponer a la agencia a una responsabilidad potencialmente ilimitada ${ }^{53}$.

Se prevé además que la violación de un deber de comportamiento por parte de la agencia de calificación debe haber incidido en el resultado del procedimiento valorativo llevado a cabo por ella misma: es decir, la conducta debe traducirse en una alteración del resultado del procedimiento de calificación, que no se habría verificado en ausencia de la transgresión. Además, es resarcible únicamente el daño que sea consecuencia de tal inobservancia. En consecuencia, debe ser probada la subsistencia de un doble nexo causal: in primis entre incumplimiento del deber de conducta debida y el juicio final de calificación, y luego, también, entre tal juicio y el daño padecido por parte del inversionista o emisor.

Así mismo, la repartición de la carga de la prueba tiene una disciplina parcial en el texto del artículo 35 bis, en particular en el inciso I, frases 2 y 3 , y en el inciso 2. Los legitimados activos tienen que demostrar la violación de uno de los deberes de comportamiento enumerados en el Reglamento y, al mismo tiempo, el doble nexo de causalidad de que viene de hablarse ${ }^{54}$. Por otra parte, los inversionistas tienen la carga de probar que su decisión de inversión, no inversión o espera fue determinada por la confianza que depositaron en lo correcto del juicio de calificación elaborado por la agencia llamada a responder.

La reglamentación europea admite además limitaciones anticipadas de responsabilidad que sean razonables y proporcionadas, fuera de que sean conformes al derecho nacional aplicable al caso concreto según las normas de derecho internacional privado pertinentes ${ }^{55}$. Todo análisis relativo a la validez y a la eficacia de tales pactos de limitación anticipada de responsabilidad entre las agencias y los inversionistas o emisores tiene que darse según el derecho aplicable de los

53 Considerando 33 del Reglamento UE n. ${ }^{\circ} 462 / 2013$.

54 Para una crítica a tal repartición de la carga probatoria ver Di Donna, Danni da rating e rimedi degli investitori, cit., 286 ss. Para una discusión sobre el tema ver, además, para el ordenamiento alemán, Schroeter, Ratings, cit., 845 ss.; Schantz, Die zivilrechtliche Verantwortlichkeit von Ratingagenturen gegenüber Investoren, Berlin, 2015,362 s., 366

55 Art. 35 bis inc. 3 Reglamento CE n. ${ }^{\circ}$ 1060/2009. 
Estados miembros. Y ello vale también para el caso, para nada infrecuente, en el que esta limitación envuelva las pretensiones resarcitorias de terceros ${ }^{5}$. De este modo el legislador europeo parece afrontar sobre el plano de la limitación anticipada de la responsabilidad el problema de la potencial extensión ilimitada de responsabilidad de las agencias de calificación. Solo en la práctica podrá evaluarse de manera definitiva si esta estrategia definitivamente resulta útil y justificada.

\section{Algunas reflexiones al margen del remedio europeo}

Estas previsiones tienen el mérito de sancionar de manera irrefutable la existencia de un derecho al resarcimiento del daño en cabeza de los inversionistas y emisores como consecuencia de la difusión en el mercado de juicios inexactos, incluso con independencia de una relación contractual. Se trata de un punto de llegada que no puede darse por descontado en el derecho de los Estados miembros, particularmente si se considera que el daño padecido por emisores o inversionistas es consecuencia de la difusión, por parte de las agencias de calificación, de informaciones inexactas a un público indistinto ${ }^{57}$.Tal daño tiene consistencia puramente financiera, ya que no es generado como consecuencia de la violación de una situación jurídica subjetiva tutelada por el ordenamiento, sino que se manifiesta únicamente en su entidad económica; toca así la ya conocida y debatida problemática de la resarcibilidad del daño meramente patrimonial $5^{8}$.

Es además de particular importancia práctica la codificación de los deberes de comportamiento cuya violación genera responsabilidad en el sentido del artículo 35 bis del Reglamento CE n. ${ }^{\circ}$ Io6o/2009, porque, por un lado, permite a las agencias conocer a priori los comportamientos que los exponen a una responsabilidad y, haciendo calculable el riesgo, lo vuelve asegurable; por otro, aclara desde un inicio a los sujetos lesionados aquello que deben probar para obtener

${ }_{5} 6$ Sobre el puto ver, con fines representativos, la cláusula insertada en las condiciones generales de contrato predispuestas por Fitch Ratings (disponible en: http://www.fitchratings.com/web/ en/dynamic/terms-of-use.jsp): "Any person or entity who uses a rating does so entirely at his, her or its own risk. Should any such person or entity be entitled to recover damages from Fitch under any legal theory, such person or entity agrees, to the extent permitted by law, that the total liability of Fitch in connection with such rating is limited to actual direct damages that can be proven up to an amount not to exceed three times the net fees received by Fitch with respect to such rating".

57 El tema de la responsabilidad por difusión de informaciones inexactas por parte de profesionales es particularmente espinoso y ha jugado un rol central en el debate que se ha llevado a cabo en la doctrina civilista italiana en relación con los confines de la responsabilidad civil. Sobre el punto ver los trabajos de Busnelli, "Itinerari europei nella 'terra di nessuno tra contratto e fatto illecito': la responsabilità da informazioni inesatte”, Contr. e impr., I99I, II, 539 ss.; CAstronovo, La nuova responsabilità civile, $3 .^{\text {a }}$ ed., Milano, 2006, 496 ss.

58 Una primera panorámica del tema en MAGGIOLO, Il risarcimento della pura perdita patrimoniale, Milano, 2003, I ss.; CAstronovo, "Del non risarcibile aquiliano: danno meramente patrimoniale, cd. perdita di chance, danni punitivi, danno cd. esistenziale", Europa e dir. priv., 2008, 3 I 5 ss.; Muccioli, "Osservazioni in tema di danno meramente patrimoniale", N. giur. civ. comm., 20o8, II, 430 ss. 
un resarcimiento. Así, por ejemplo, el deber de divulgar al público los métodos, modelos e hipótesis fundamentales utilizados, que deben ser rigurosos, sistemáticos, continuativos y sujetos a convalidación sobre la base de la experiencia histórica ${ }^{59}$, asume un relieve determinante en el proceso de determinación de la responsabilidad de las agencias de calificación por la difusión de una calificación inexacta ${ }^{60}$.

Por otra parte, al delinear el estándar de diligencia que las agencias deben respetar en el desarrollo de su actividad, contienen criterios de juicio que adquieren relevancia en todas las valoraciones de su actuar, también con referencia a la responsabilidad de las agencias según el derecho de los Estados miembros. Desarrollan así una tarea de relevancia central en el contexto del doble marco de responsabilidad que fue delineado por la normativa europea. Es evidente que la enucleación de los deberes comportamentales previstos por la disciplina europea resulta determinante en el análisis de la responsabilidad civil de las agencias.

De las reglas apenas descritas se pueden desprender vínculos relevantes con respecto a la responsabilidad de las agencias. Por un lado, el hecho de que el criterio de culpabilidad requerido se haya fijado en el dolo o en la culpa grave lleva consigo sin lugar a dudas dificultades no indiferentes en la asignación de la carga probatoria correspondiente. Por otro, la necesidad para los lesionados de probar la existencia de un doble nexo de causalidad agrava ulteriormente su posición bajo un perfil procesal. Fuera de ello, no todas las conductas codificadas en el Anexo iII del Reglamento sobre la calificación resultan idóneas, incluso solo en abstracto, para influenciar el resultado del procedimiento de calificación: la ausencia, por ejemplo, de "procedimientos administrativos o contables sólidos" de acuerdo con el Anexo III, sección I, n. ${ }^{\circ}$ I 2 del Reglamento puede difícilmente ser idónea para influenciar el resultado del procedimiento de calificación. De todos estos elementos emerge entonces cómo la base normativa europea no resulta en su conjunto particularmente favorable a las víctimas del daño.

Por otra parte, parece evidente a primera vista que la disciplina que viene de describirse no está en capacidad de resolver eficazmente la cuestión de la responsabilidad de las agencias de calificación, a menos que haya una adecuada labor de interpretación. La complejidad y las peculiaridades de las nuevas disposiciones han sido puestas de relieve por los intérpretes que en particular han subrayado los grandes impases que se derivan de la elección de no uniformar completamente la disciplina de la responsabilidad en el plano europeo, sino de delegar una parte bastante relevante de ella a los varios derechos nacionales, y al mismo tiempo, de mantener en vida, fuera del marco europeo, todas las pretensiones resarcitorias previstas por los derechos nacionales. Se introdujo así una doble vía: por un lado, la responsabilidad regulada por la disciplina europea; por otro,

6o Sobre el tema ver Di Donna, La responsabilità civile delle agenzie di rating, cit., I 77 ss. 
la responsabilidad sujeta a las previsiones del derecho nacional. En un ámbito como el de la calificación, que por naturaleza se desarrolla en un plano transnacional, esta fragmentación de la disciplina resulta particularmente problemática.

No se puede dejar de lado que la actividad de las agencias de calificación afecta a diversos interlocutores, como pone de relieve el Reglamento mismo. Ella involucra, por un lado, los intereses de los emisores que son objeto -ellos mismos en su entidad económica o sus emisiones- de las evaluaciones llevadas a cabo por las agencias con referencia a su solidez y solvencia; y por otro, los intereses de la generalidad de los inversionistas, que tienen en cuenta la calificación como el dato informativo más importante en su elección de inversión. Se trata de intereses en cabeza de sujetos que actúan en los mercados financieros, uno frente al otro, y que con respecto a la calificación tienen prospectivas diametralmente opuestas.

Los emisores apuntan a tener la calificación más alta posible, que les garantice disponer de mejores condiciones de acceso al crédito y que haga que los instrumentos financieros emitidos lleguen a un público de inversionistas más amplio y a un precio de mercado más elevado. Una calificación erróneamente muy baja es por lo tanto lesiva de sus intereses patrimoniales ya que conduce, por ejemplo, a una mayor dificultad de acceso a préstamos y a una disminución del valor de sus emisiones en el mercado. Una calificación erróneamente muy optimista es, por contrario, lesiva de los intereses patrimoniales de los inversionistas que han confiado en ella debido a la profesionalidad y buena reputación de las agencias. Tal daño puede manifestarse in primis como una remuneración del capital invertido en una menor medida con respecto a las expectativas generadas por el alto nivel de la calificación, pero también como pérdidas y depreciaciones no pronosticadas.

Así las cosas, si las agencias de calificación actúan en varias esferas con intereses contrapuestos, sería de esperarse que también la regulación de la actividad de las agencias, e in primis su responsabilidad, estuviera netamente diferenciada con base en los diferentes supuestos y sujetos implicados. Este es el tipo de aproximación prevaleciente entre los estudiosos que analizan las pretensiones previstas por los diferentes ordenamientos nacionales.

Pese a la introducción de una nueva disciplina de la actividad de calificación por parte del legislador europeo, materializada en el Reglamento CE $n .^{\circ}$ I060/2009, y en particular luego del delineamiento de una base normativa explícita para la responsabilidad de las agencias de calificación por cuenta del segundo reglamento modificatorio, es necesario un cambio de perspectiva. Las nuevas reglas prevén una disciplina unitaria para la actividad de las agencias de calificación y una única responsabilidad en caso de violación de las normas de comportamiento impuestas, que lacónicamente implican la misma disciplina para los daños ocasionados a emisores e inversionistas, prescindiendo del hecho de que la evaluación haya sido erradamente o muy optimista o muy pesimista, 
y por lo tanto prescindiendo de los sujetos que en concreto resultan lesionados por la inexactitud de las evaluaciones sobre el mérito del crédito difundidas en el mercado.

Un cambio de perspectiva en la reglamentación de la materia tratada incita a una dirección diversa de su estudio. La aproximación monista elegida por el derecho de la Unión Europea, que prescinde de la pluralidad de los interlocutores a quienes va dirigida la actividad de la calificación, impone el análisis de la actividad de las agencias de calificación en la pluralidad de direcciones en que ella se desarrolla.

Y no se puede descuidar que las relaciones con tales interlocutores pueden configurarse de diversas formas: los emisores pueden encargar a las agencias de calificación la elaboración de una valoración global de la propia solvencia, o una valoración limitada a una cierta emisión, instaurando con ellas una relación contractual (el llamado solicited rating), o en cambio pueden ser tomados como objeto de análisis con prescindencia de su consentimiento (el llamado unsolicited rating). A su vez, también los inversionistas pueden estipular con las agencias de calificación un contrato para obtener una valoración del mérito crediticio de un ente (piénsese en particular en los inversionistas institucionales) o pagar una suscripción para recibir noticias sobre las clasificaciones, o pueden informarse aliunde -a través de prospectos informativos y documentos de oferta, o autónomamente- acerca de las evaluaciones hechas por las agencias de calificación y utilizarlas como referencia para sus decisiones de inversión, prescindiendo de cualquier relación anterior con las agencias mismas. Es evidente que se trata de situaciones que, desde un punto de vista fáctico y desde uno jurídico, son muy diferentes entre ellas y deben ser tratadas por separado, en particular porque el problema de la resarcibilidad del daño meramente patrimonial se presenta tan solo en el plano de la responsabilidad extracontractual.

Las peculiaridades que distinguen los supuestos considerados por la normativa europea necesariamente tienen relevancia central en el delineamiento del régimen de responsabilidad al que resultan atadas las agencias de calificación. Por tanto, es indispensable analizar singularmente las posibles combinaciones, sea con miras al encuadramiento de la pretensión europea en los ordenamientos nacionales, sea para individuar las pretensiones de derecho nacional salvaguardadas por el artículo 35 bis, inciso 5 del Reglamento CE n. ${ }^{\circ}$ Io6o/2009, para poder traer a la luz las diferencias en la disciplina que, en el doble marco de responsabilidad que se ha venido delineando en este ámbito, resultan necesarias por los elementos de cada caso en concreto.

Es además indispensable traer a la luz las especificidades que la responsabilidad civil presenta en el derecho de los mercados financieros, en primer lugar cuando ella se refleja en la responsabilidad civil de los llamados reputational intermediaries. Toda reflexión relativa a la responsabilidad de tales sujetos se encuentra con problemáticas de eficiencia económica, por ejemplo con la preocupación de 
que una responsabilidad muy dilatada, y condenas resarcitorias muy conspicuas, puedan conducir a una salida de algún operador del mercado ${ }^{6 \mathrm{I}}$, con el temor de que ello pueda llevar a un aumento de los costos de la calificación, en daño de la generalidad de los asociados ${ }^{62}$, con el peligro de un "deep pocket syndrome" 3 o incluso con la aprensión -en realidad más ideológica que real ${ }^{64}$ - de que un régimen más riguroso se traduzca en overdeterrence, influenciado así en manera negativa las valoraciones del mérito crediticio efectuadas ${ }^{65}$.

También con referencia a la cuestión de la responsabilidad de las agencias de calificación, se debe encontrar un justo balance entre dos exigencias contrapuestas e igualmente relevantes: por un lado, la necesidad fundamental, común a los otros supuestos de responsabilidad que se insertan en el contexto de los mercados financieros, de garantizar una tutela adecuada de los emisores evaluados y de los inversionistas que han confiado en los juicios expresados bajo la forma de la calificación; por otro lado, la necesidad de asegurar un funcionamiento eficiente del mercado de la calificación del crédito. Emergen nuevamente las peculiaridades que caracterizan la responsabilidad de los sujetos que operan en los mercados financieros y las funciones específicas de la responsabilidad civil por el resarcimiento del daño que se verifica como consecuencia de actividades desarrolladas en tales mercados.

Ya que el legislador europeo desechó la idea -fuerte en el debate relativo a la responsabilidad de los 'intermediarios reputacionales' ${ }^{\text {'6 }}$ - de introducir un mon-

6I Sobre el punto ver Ponzanelli, "Responsabilità civile e mercato finanziario", Danno e resp., 2002, 98 .

62 Parmeggiani, "Some Rating Failures and Several Regulatory Weaknesses: The US and the EU Perspectives", Le agenzie di rating, al cuidado de Principe, cit., 87.

63 Con referencia a un ejemplo distinto de 'intermediario reputacional', en particular, los auditores, ver Montalenti y Balzola, "La società per azioni quotata", Le nuove s.p.a., dirigido por Cagnasso y Panzani, vol. II, Bologna, 20 Io, I983; BALzola, "La responsabilità contrattuale del revisore contabile: obbligazione di mezzi o di risultato?", Giur. it., 2006, 2334 ss.; Montalenti, "La società quotata", Trattato di diritto commerciale, vol. Iv.2, Cotrino (ed.), Padova, 2004,3 I 2. Véase además en el plano internacional Flores, "New Trends in Auditor Liability", European Business Organization Law Review, I 2, 201 I, 426, 43 I s.

64 En este sentido Libertini y Fаввіо, "Concorrenza e rating finanziario", Le agenzie di rating, Principe (ed.), cit., I6r.

65 Picciau, La responsabilità delle agenzie di rating per la diffusione di giudizi inesatti sul mercato finanziario, cit., I.

66 Para un ejemplo de limitación de la responsabilidad a un máximo predeterminado por el legislador para un intermediario reputacional distinto ver, en el ordenamiento alemán, el $\$ 323$ inc. 2 HGB que limita la responsabilidad de los auditores en caso de culpa (pero no de dolo) en la revisión de sociedades con cotización en bolsa a un máximo de cuatro millones de euros. Para un primer comentario al respecto ver Евке, "Commento al \$323 нав", Münchener Kommentar zum Handelsgesetzbuch, Schmidt (ed.), vol. Iv, 3. ${ }^{a}$ ed., München, 20I3, Rdnr. I ss.; Habersack y SchürnnRand, “Commento al $\$ 323$ HGB”, Handelsgesetzbuch. Großkommentar, fundado por Staub, vol. viI.I, 5. ${ }^{a}$ ed., Berlin, 20IO, 222 ss.; Kremer, Die zivilrechtliche Verantwortlichkeit des Wirtschaftsprïfers gegenüber Kapitalanlegern, Göttingen, 2007,32 ss., 204 ss.; STRASSER, Die Haftung der Wirtschaftsprïfer gegenüber Kapitalanlegern für feblerbafte Testate, Frankfurt a.M., 
to máximo al quantum resarcitorio al que pueden resultar obligadas las agencias de calificación (el llamado cap $)^{67}$, se hace necesaria la búsqueda de otros instrumentos, compatibles con el texto normativo del Reglamento europeo (o deducibles del derecho nacional), dirigidos a garantizar un equilibrio entre los diversos intereses en juego.

Esta justa ponderación debe buscarse no solo bajo el perfil del encuadramiento jurídico de la pretensión de derecho europeo en el cauce de la responsabilidad contractual o extracontractual, con todas las consecuencias relevantes que deriven de tal elección bajo el perfil del régimen de responsabilidad aplicable $^{68}$, sino también al delinear los diversos elementos de la responsabilidad, cuya definición se solicita a los legisladores nacionales. Incluso al interior de los límites impuestos por el legislador europeo, pareciera que todavía queda un amplio margen de maniobra.

\section{Bibliografía}

Armour y McCahery, "After Enron: Improving Corporate Law and Modernizing Securities Regulation in Europe and in the US", Amsterdam Center for Law \& Economics Working Paper No. 2006-07, I ss.

Avgouleas, "The Global Financial Crisis - Behavioural Finance and Financial Regulation - In Search of a New Orthodoxy", f. Corp. L. Studies 9, 2009, 33 ss.

Balzola, "La responsabilità contrattuale del revisore contabile: obbligazione di mezzi o di risultato?", Giur. it., 2006, 2334 ss.

Bar-Gill, "The Law, Economics and Psychology of Subprime Mortgage Contracts", Cornell L. Rev. 94, 2009, 1073 ss.

2003, 7 ss.; Schiemann, "Wandlungen der Berufshaftung", Festschrift für foachim Gernhuber zum 7 o. Geburtstag, Lange et al. (eds.), Tübingen, I 993,387 ss. Es así como el legislador alemán dio cumplimiento a las indicaciones contenidas en la Recomendación 2008/4773/CE relativa a la limitación de la responsabilidad civil de los auditores legales y de las empresas de revisión contable (sobre la cual no intervino la amplia reforma de la materia que terminó en 20I4) que sugirió a los Estados miembros, entre otras, la "fijación de un importe financiero máximo o de una fórmula que permita el cálculo de tal importe". No puede obviarse que en materia de responsabilidad de auditores frente a la disciplina dictada con reglamento en materia de agencias de calificación la legislación europea es bastante más blanda, al estar contenida en una simple recomendación. Sobre el tema ver Flores, New Trends in Auditor Liability, cit., 4I 5 ss.

67 En este sentido ver, ej., Parmeg giani, Some rating failures and several regulatory weaknesses, cit., 87.

68 En particular, se derivaría una ampliación del término de prescripción, una repartición de la carga probatoria más favorable a la parte lesionada. Para una revaluación de esta opinión, común en la doctrina y subyacente en muchas decisiones jurisprudenciales, ver el puntual análisis de MAgGIolo, Il risarcimento della pura perdita patrimoniale, cit., IO2 ss., espec. I I 5 ss. 
Blaurock, "Verantwortlichkeit von Ratingagenturen - Steuerung durch Privatoder Aufsichtsrecht?", ZGR, 2007, 642 ss.

Buonocore, "Etica degli affari e impresa etica”, Giur. comm., 2004, i8 I ss.

Busnelli, "Itinerari europei nella 'terra di nessuno tra contratto e fatto illecito': la responsabilità da informazioni inesatte”, Contratto e impresa, I99I, II, 539 ss.

Calabresi, The Costs of Accidents, A Legal and Economic Analysis, New Haven, i 970.

Carriero, "Previsioni, opinioni, certezze: le agenzie di rating", Europa e dir. priv., 2012,873 ss.

Castronovo, "Del non risarcibile aquiliano: danno meramente patrimoniale, cd. perdita di chance, danni punitivi, danno cd. esistenziale”, Europa e dir. priv., 2008, 315 ss.

Castronovo, La nuova responsabilità civile, $3 \cdot{ }^{\mathrm{a}} \mathrm{ed} ., \mathrm{M}$ Milano, 2006.

Cazorla Prieto, "La inserción de las agencias de 'rating' en el incipiente gobierno de la globalización financiera”, Derecho Bancario y Bursátil, n. ${ }^{\circ}$ I 29, 20I3, 206 ss.

Cervone, "Riflessioni per un'indagine sulle agenzie di rating", Riv. trim. dir. econ., 2009, I, 293 ss.

Coffee, Gatekeepers. The Professions and Corporate Governance, Oxford, 2006.

Choi, "Market Lessons for Gatekeepers", Nw. U.L. Rev., 92, I998, 9 I6 ss.

Crawford, "Hitting the Sweet Spot by Accident: How Recent Lower Court Cases Help Realign Incentives in the Credit Rating Industry", Conn. Law Rev., 42, 2009, I 8 ss.

Darbellay y Partnoy, “Credit Rating Agencies and Regulatory Reform”, Research Handbook on the Economics of Corporate Law, Claire A. Hill y Brett H. McDonnell (eds.), Cheltenham (UK) y Northhampton (USA), 20 2 2, 290 ss.

Di Donna, "Danni da rating e rimedi degli investitori", Le agenzie di rating, Principe (ed.), Milano, 20I4, 275 ss. 
Di Donna, La responsabilità civile delle agenzie di rating, Padova, $20 \mathrm{I} 2$.

Ditrrich, The Credit Rating Industry. Competition and Regulation, Köln, 2007.

Drigo, "La responsabilità delle agenzie di rating per il danno all'informato. L'esperienza statunitense", Rass. dir. civ., 2006, 490 ss.

Евке, “Commento al $\$ 323$ няв”, Münchener Kommentar zum Handelsgesetzbuch, Schmidt (ed.), vol. Iv, 3. ${ }^{a}$ ed., München, 20I3, Rdnr. I ss.

FACCI, "Le agenzie di rating e la responsabilità per informazioni inesatte", Contratto e impresa, 2008, I, I64 ss.

Ferri y Lacitignola, Le agenzie di rating, 2. ${ }^{\text {a }}$ ed., Bologna, 2014.

Flores, "New Trends in Auditor Liability", European Business Organization Law Review, I 2, 20I I, 426 ss.

García Alcubilla, "La supervisión de las agencias de rating en Europa", Derecho Bancario y Bursátil, n. ${ }^{\circ}$ I 29, 2013 , 2 I 9 ss.

Gilson, "Value Creation by Business Lawyers: Legal Skills and Asset Pricing", Yale L. 7., 94, 1984, 239 ss.

Gilson y Kraakman, "The Mechanisms of Market Efficiency”, Va. L. Rev. 70, I984, 620 ss.

Grundmann, Hofmann y Möslein, "Finanzkrise und Wirtschaftsordnung: Krisenursachen, Finanzmarktstabilisierung, Finanzmarktstabilität", Finanzkrise und Wirtschaftsordnung, Grundmann, Hofmann y Möslein (eds.), Berlin, 2009.

HaAR, "Das deutsche Ausführungsgesetz zur EU-Rating-Verordnung - Zwischentappe auf dem Weg zu einer europäischen Finanzmarktarchitektur", ZBB, 2009, I 78 ss.

HaAR, "Die Rolle der Ratingagenturen bei der Corporate Governance von Banken", Handbuch Corporate Governance von Banken, Норт у WohlmannsTetter (eds.), München, 20 I I, 233 ss.

HaAR, "Neues zur Haftung von Ratingagenturen im Zuge der zweiten Novelle der Rating-Verordnung (CRA III)?”, DB, 20I3, 2492 ss. 
Habersack, "Rechtsfragen des Emittenten-Ratings", zHR, I69, 2005, I89 ss.

Habersack y Schürnbrand, “Commento al $\$ 323$ hgb”, Handelsgesetzbuch. Großkommentar, fundado por Staub, vol. vir. I, 5. ${ }^{\mathrm{a}}$ ed., Berlin, 2010, 222 ss.

Hill, "Limits of Dodd-Frank's Rating Agency Reform", Chapman L. Rev., I 5, 201 I, I43 ss.

Hunt, "Credit Rating Agencies and the 'Worldwide Credit Crisis': The Limits of Reputation, The Insufficiency of Reform and a Proposal for Improvement”, en Colum. Bus. L. Rev. I09, 2009, I ss.

Husisian, "What Standard of Care Should Govern the World's Shortest Editorials?: An Analysis of Bond Rating Agency Liability", Cornell L. Rev., 75, 4IO, I990, 430.

Kraakman, "Corporate Liability Strategies and the Costs at Legal Controls", Yale L. F., 93, 1984, 857 ss.

KraAkman, “Gatekeepers: The Anatomy of a Third-Party Enforcement Strategy”, 7. L. Econ. \& Org., 2, I986, 53 ss.

Kremer, Die zivilrechtliche Verantwortlichkeit des Wirtschaftsprüfers gegenüber Kapitalanlegern, Göttingen, 2007.

Kumpan, "Regulierung von Ratingagenturen - ein anreizorientierter Ansatz", Festschrift für Klaus 7. Hopt zum 7o. Geburtstag, vol. II, Grundmann et al. (eds.), Berlin, 2010, 2 I 57 ss.

Leyens, "Intermediary Independence: Auditors, Financial Analysts and Rating Agencies", Journal of Corporate Law Studies, 201 I, 33 ss.

Libertini y Fаввio, "Concorrenza e rating finanziario", Le agenzie di rating, Principe (ed.), Milano, 20i4, 26 I ss.

Lipsey y Lancaster, "General Theory of Second Best", Rev. Econ. Stud. 24, I956, I I ss.

López Martínez, "Independencia y conflictos de interés en la actividad de las agencias de rating", Derecho Bancario y Bursátil, n. ${ }^{\circ}$ I 29, 201 3, 238 ss.

MAGgiolo, Il risarcimento della pura perdita patrimoniale, Milano, 2003. 
Montalenti, "Le agenzie di rating: appunti”, Giur. comm., 20I3, I, 5 I 4 ss.

Montalenti, "La società quotata", Trattato di diritto commerciale, vol. Iv.2, CoTTINo (ed.), Padova, 2004, 3 I 2 ss.

Montalenti y Balzola, "La società per azioni quotata”, Le nuove s.p.a., Cagnasso y Panzani (dirs.), vol. ir, Bologna, 20 io, i983.

Muccioli, "Osservazioni in tema di danno meramente patrimoniale", N. giur. civ. comm., 2008, II, 430 ss.

Muñoz García, "Los rating no solicitados”, Derecho Bancario y Bursátil, n. I 29, 2013,246 ss.

Olivieri, "I servizi di rating tra concorrenza e regolazione", Analisi giur. econ., 2012,285 ss.

Parmeggiani, "La regolazione delle agenzie di rating tra tentativi incompiuti e prospettive future", Giur. comm., 2010, I, I 35 ss.

Parmeggiani, "Some Rating Failures and Several Regulatory Weaknesses: the US and the EU Perspectives", Le agenzie di rating, Principe (ed.), Milano, 20I4, 75 ss.

Partnoy, "Overdependence on Credit Rating Was a Primary Cause of the Crisis”, San Diego Legal Studies o9/o I 5, 2009, 5 ss.

Perrone, Informazione sul mercato e tutele dell'investitore, Milano, 2003.

Picciau, La responsabilità delle agenzie di rating per la diffusione di giudizi inesatti sul mercato finanziario, Università degli Studi di Milano, tesis doctoral, 20 I32014.

Pinto, "Control and Responsibility of Credit Rating Agencies in the United States", Am. 7. Comp. L., 54, 2006, 343 ss.

Ponzanelli, "Responsabilità civile e mercato finanziario", Danno e resp., 2002, 98.

Presti, s.v. “rating”, Enc. dir., Ann. vii, Milano, 20I4, 847 ss.

Presti, "Le agenzie di rating: dalla protezione alla regolazione", $7 u s, 2009,65$ ss. 
Presti, “Take the aAA train: note introduttive sul rating”, Analisi giur. econ., 20 I 2 , 25 I ss.

Rousseau, "Enhancing the Accountability of Credit Rating Agencies: The Case for a Disclosure-Based Approach”, McGill L. F., 5 I , 2006.

Roтт, "Europäisierung des Rechts der Finanzintermediäre”, EWS, 2008, 2 I ss.

Scaroni, "La responsabilità delle agenzie di rating nei confronti degli investitori”, Contratto e impresa, 201 I, 3, 764 ss.

Schantz, Die zivilrechtliche Verantwortlichkeit von Ratingagenturen gegenüber Investoren, Berlin, 2015 .

Schäfer, "Strafe und Prävention im Bürgerlichen Recht”, AcP, 202, 2002, 399 ss.

Schiemann, "Wandlungen der Berufshaftung", Festschrift für Foachim Gernbuber zum 7o. Geburtstag, LANGE et al. (eds.), Tübingen, I993, 387 ss.

Schroeter, Ratings - Bonitätsbeurteilungen durch Dritte im System des Finanzmarkt-, Gesellschafts- und Vertragsrechts, Tübingen, 2014.

Schuler, Regulierung und zivilrechtliche Verantwortlichkeit von Ratingagenturen, Frankfurt a.M., 2012 .

Schwarcz, "Private Ordering of Public Markets: The Rating Agency Paradox", U. Ill. L. Rev. I4, 2002, I ss.

Schwarcz, "Protecting Financial Markets: Lessons from the Subprime Mortgage Meltdown”, Minnesota Law Review, 93, 2008, 373.

Shapiro, "Premiums for High Quality Products as Returns to Reputations", Quart.7. Econ. 98, I983, 659 ss.

STrasser, Die Haftung der Wirtschaftsprüfer gegenüber Kapitalanlegern für feblerbafte Testate, Frankfurt a.M., 2003.

Sylla, "A historical Primer on the Business of Credit Rating", Ratings, Rating Agencies and the Global Financial System, Levich, Majnoni y Reinhart (eds.), New York, 2002. 
Tapia Hermida, "Novedades en la regulación comunitaria y española de las Agencias de Calificación Crediticias (Agencias de rating)", Derecho Bancario y Bursátil, n. ${ }^{\circ}$ I 23, 201 I, 296 ss.

Turner, The Turner Review 2009 - A Regulatory response to the Global Financial Crisis, disponible en: http://www.fsa.gov.uk/pubs/other/turner_review.pdf

WAGNER, "Die Haftung von Ratingagenturen gegenüber dem Anlegerpublikum”, Einheit und Vielheit im Unternehmensrecht, Festschrift für Uwe Blaurock zum 70. Geburtstag, Jung, Lamprecht et al. (eds.), Tübingen, 2013.

Wagner, "Prävention und Verhaltenssteuerung durch Privatrecht - Anmaßung oder legitime Aufgabe?", $A c P$, 206, 2006, 363 ss. 